\title{
Monitoring of Physiological Signs and Their Impact on The Covid-19 Pandemic: Review
}

\author{
Abdoullah Bella, Rachid Latif, Amine Saddik, Fatima Zahra Guerrouj \\ LISTI, ENSA Ibn Zohr University Agadir, 80000, Morocco \\ bellaabdoullah@gmail.com
}

\begin{abstract}
Keywords: Physiological signs, Heart rate, Respiratory rate, Monitoring, Non-contact techniques, Image processing, RGB camera, Covid-19.

Abstract: $\quad$ Recently, monitoring of physiological signs such as heart rate and respiratory rate is very important, especially when we are talking about pandemics like Covid-19. In this paper we present a state of the art on the different techniques used for heart rate and respiratory rate extraction. These techniques presented will be based on image processing, were traditional sensor-based techniques creating a lot of problem at the contact level between patient and doctor. For this reason, we focus on non-contact techniques to avoid these problems. Generally, the literature review shows that non-contact monitoring techniques are based on RGB, thermal and multispectral cameras, the choice between these different cameras depends on the application that will be used. For example, thermal cameras are dedicated to the prediction of respiratory rate and temperature, while RGB and multispectral cameras are used for heart rate.
\end{abstract}

\section{INTRODUCTION}

The human race has survived many contagious pandemics, from smallpox, pass-ing by the Measles, Viral haemorrhagic fevers,Severe acute respiratory syndrome, H5N1, Spanish flu, arriving to the pandemic that has moved all communities today which is the Covid-19[1-2]. The common point between all these diseases is that all those pandemics were contagious, with different degrees of risk. Healthcare system in such cases should be based on non-contact approaches to avoid any influence. That's exactly why from years ago to now days, the world was merging into monitor-ing, consequently, remote healthcare is an emerging research field as it is sensible [3-4].

From years ago, embedded systems and biomedical engineering have been shown a very major role in responding to biomedical problems. Now a days, this role be-comes more important than any other time due to what the world is living today be-cause of the pandemic Covid-19 that has spread globally, but what is sure is this pandemic is not the only one that affects heart rate, temperature, and respiratory rate which should be monitored by a medical team, in order to be ready for any interven-tion [5-6].
Heart rate and respiratory rate monitoring have been presented a very vast inter-rogation, especially when it comes to a contagious disease patient, that should be observed with non-contact tools, and that's for sure what introduces image pro-cessing devices. In like manner, many non-contact approaches were developed in this context, and showed good results, standing on image processing, artificial intelligence or other [7-8]. After studying the human body, we realized that it is extremely possi-ble to sum up its impulsions from the skin, it's like if our body communicates with our extern parts. To illustrate these impulsions, many methods and approaches were proposed, starting by colour-based methods, arriving to motion-based ones [9-10].

The work presented in this paper is based on an overview of the different contact-less techniques to get respiratory and cardiac frequency. The paper is divided into four sections, after the introduction, we have state of art, in this part we will treat the applications used and the tools for contactless diagnostics. The 3rd part is reserved for the discussion and proposed solution and finally the paper ends with a conclusion. 


\section{State of the art: the latest developments}

We PPG also call Photoplethysmography is a low-cost non-invasive tool, that was pro-posed for the first time by Hertzman et. al 1937. This technique is a very known used technique in biomedical engineering which gives good result in measuring $\mathrm{HR}, \mathrm{RR}$ and $\mathrm{SpO} 2$, thanks to its sample using manner and low-cost. We find also the iPPG technique that is the amelioration of the classical PPG technique.

The iPPG model is mainly based on three sections. The first one is "light source", then a "human skin" and finally a "video camera". The light source illuminates human skin, then the camera captures the colour changes [11]. The figure 1 shows the skin reflection mod-el of imaging photoplethysmography (iPPG) [ 12].

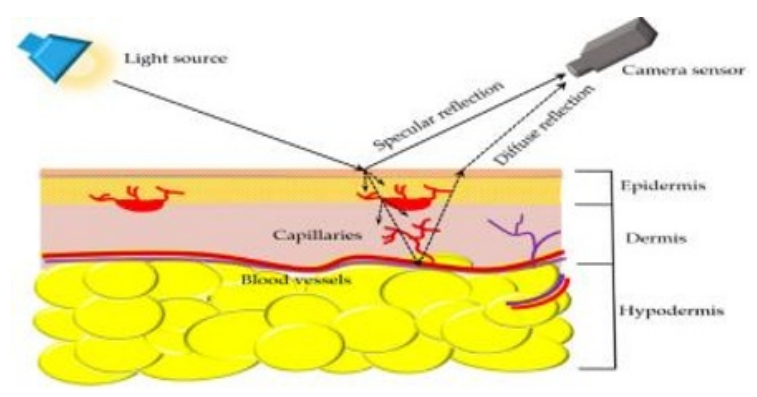

Figure 1: Skin reflection model of imaging photoplethysmography (iPPG) [12]

Mathematically, we can represent the light that's been reflected from the skin as the follow formula [12]:

$$
\mathrm{Pk}(\mathrm{t})=\mathrm{I}(\mathrm{t})(\mathrm{Rs}(\mathrm{t})+\mathrm{Rd}(\mathrm{t}))+\mathrm{Rn}(\mathrm{t})
$$

Where:

- $\quad P k(t)$ : the RGB channels of the skin pixel

- $\quad \mathrm{I}(\mathrm{t})$ : the illumination intensity level

- $\quad \operatorname{Rs}(\mathrm{t})$ : the specular reflection

- $\quad \mathrm{Rd}(\mathrm{t})$ : the diffuse reflection reflection

$\mathrm{I}(\mathrm{t})$ : modulated by both specular and diffuse

- $\operatorname{Rn}(\mathrm{t})$ : the quantization noise of the camera sensor.
S. Sanyal et al, in their work appeared in 2018, provides an approach for heart rate and respiratory rate remote monitoring, all based on measuring iPPG using Hue (range 0-0.1) and not RGB channel, thanks to its good accurate comparing to HR/Green or $\mathrm{RR} / \mathrm{Green}$. The algorithm was tested on a human face video frames of 20 seconds at $30 \mathrm{fps}$, and applied only on 10 frames, by using one image from every three successive frames, because this technique didn't affect the results, especially that 2 complete breaths are achieved within a period $\geq 20$ seconds. The algorithm starts with detecting the face and eyes using the Haar Cascade Based detection function standing on OpenCV from every frame, and converted from RGB pixels into HSV, in order to get Hue values of the pixels [13].All the steps mentioned before, are considered as a pre-processing global step for the algorithm, then comes the step of computing the average of Hue in each processed frame for the forehead. Assuming that the region of the front is the one where we can measure arterial pulsations, which finally gives as the raw iPPG signal, followed by applying a band pass filter and con-verting time series data to the appropriate frequency spectrum. The orders used for the filter differs from Heart rate to Respiratory rate, for the HR, it is 20 , while for RR it is 8 . The algorithm illustrated in the figure 2 summarizes the work proposed in the paper [13].

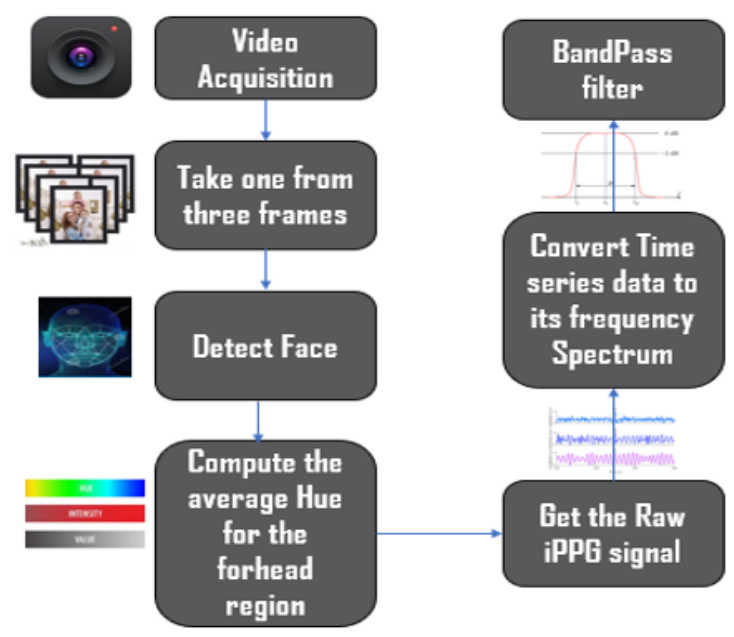

Figure 2: Block diagram of the proposed approach in [13]. 
To approve the results, the experiment of this wok was applied on 25 persons, from different skin colours, different origins and genders. For each person, two videos have been recorded to obtain at the end 50 videos. For every person of this experiment, they gave standard instruments like pulse oximeters (HR) to compare the results between the videos recorded and the proposed instrument [13].

While in the proposed study of F.T.Z. Khanamet al 2019, we find a summary of the known used methods in remote monitoring of vital signs [14]. Video camera-based methods are standing on image processing to get vital signs, and they usually use the followed framework to proceed their work [14]. The figure 3 shows the block diagram of proposed algorithm based on non-contact monitoring heart rate system.

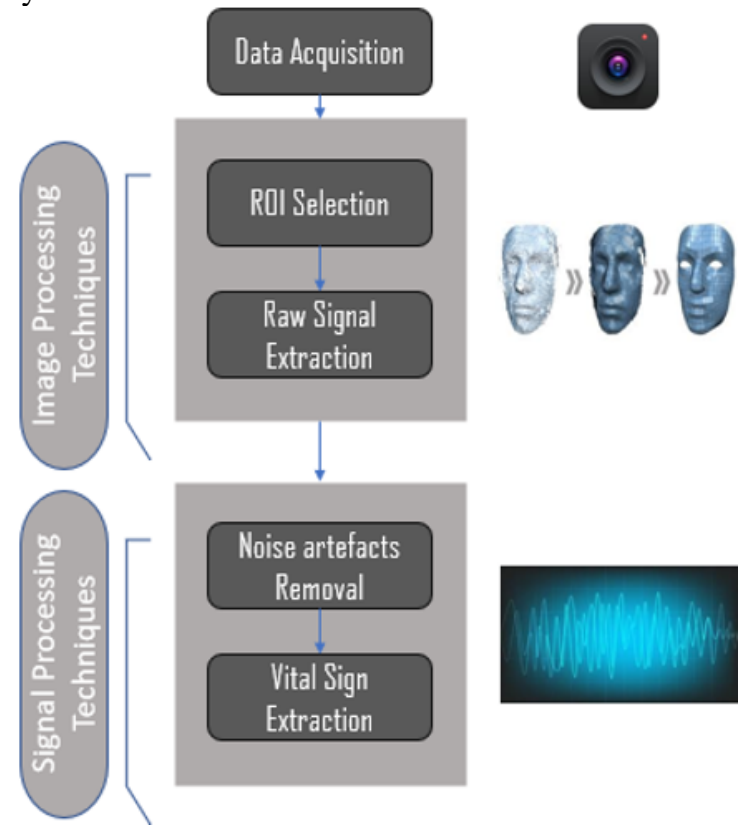

Figure 3: Block diagram of contactless monitoring system [13].

This block diagram contains some important steps, starting by the image acquisition block, which can be dealer using a Smartphone, camera, or even UAVs, the essential thing is to capture the human body to get information to be treated in our image processing algorithm. Then comes image processing technique step, which starts with the Region of Interest (ROI) selection is about selecting the region of interest, such as the forehead, face, eyes, etc. The essential thing is to attract and detect the region we are going to get pulsations from. Then comes the raw signal extraction, which is a value computes by calculating the average of pixels in the ROI area, by the following equation:

$$
\operatorname{Ir}(t), \operatorname{Ig}(t), \operatorname{Ib}(t)=\frac{\sum x, y \in R O I I(x, y, t)}{|R O I|}
$$

Where:

- $\operatorname{Ir}(t), \operatorname{Ig}(t), \operatorname{Ib}(t)$ : Three signal sources from red, green and blue components.

- $I(x, y, t):$ The brightness pixel value at image location $(\mathrm{x}, \mathrm{y})$ at time $\mathrm{t}$.

- | ROI| the size of the selected ROI.

After we got the signal, comes the step of signal processing techniques, starting by Noise Artefacts Removal step, this step aims to remove all the noise we got, due to any camera motion, skin tone or even illumination changes. After that we proceed to the vital sign extraction which is the main step, we are looking for by using frequency analysis or peak detection. Another used technique such is mentioned in [14], is the motion-based method also standing on image processing to avoid contact with the patient. This method depends on the human body motion, or at least the ROI motion.

In this context a lot of non-contact methods exist in the monitoring heart rate field, based on magnetic induction, the Doppler effect based on detecting subtle chest movements and getting vital signs standing on doppler radar [15-16], thermal imaging as in [17-18] and video camera imaging, which are a very useful means of remote monitoring of vital signs.

A lot of papers were elaborated in this context, such as the authors in [19] who proposed a non-contact processing on optical flow analysis to extract Respiratory Rate from the video of whole-body motion captured by a camera. Also, in [20], another optical flow analysis-standing on RR monitoring was presented using videos of a ROI which is the chest.

In like manner, Al-Naji et al 2016, came with a similar concept, to detect and measure Respiratory Rate with a non-contact approach, as well as respiratory cycle timing parameters by using a video camera from the movement of the chest. The figure 4 illustrates the approach proposed in [21]: 


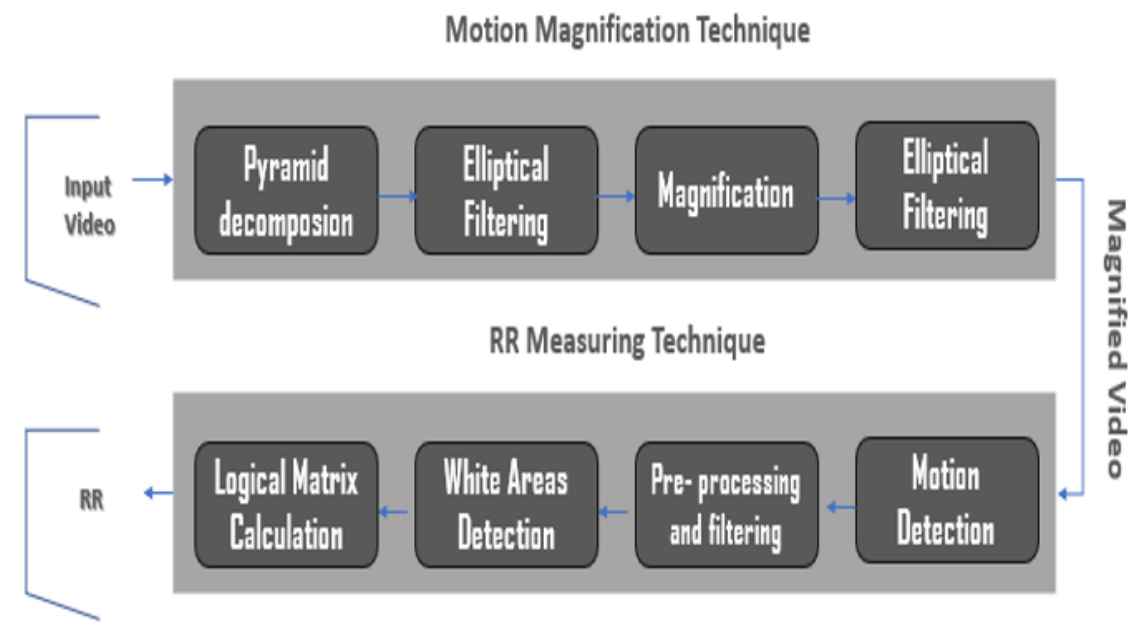

Figure 4: System diagram of the remote respiratory monitoring system [21].

\section{Discussion and proposed solution}

The use of embedded systems in the biomedical field gives flexibility to the application level. The use of embedded systems in the medical field remains limited, for this reason, we have a large field of research to explore. The embedded systems that we can use in this context can be divided into two parts, low-cost systems with limited features or systems with high computing power such as heterogeneous systems [22]. For image collection tools to estimate heart and respiratory rates, we find RGB and multispectral cameras. The difference between these two cameras is based on the desired number of bands, we can also find the use of multispectral cameras in plant surveillance in the field of precision agriculture [23].

In the same way, the authors in [24] presents a combined visible and thermal im-age processing approach using an IRT system equipped with a complementary met-al-oxide-semiconductor (CMOS) camera. This system has recorded both visible and thermal images at a speed of 30 images per second and at a resolution of $640 \times 480$ pixels, in order to acquire various vital signals, such as body temperature and heart and breathing rates from facial images, thereby allowing for the rapid and accurate identification of individuals suspected of carrying infectious diseases.However, the breathing rates are measured by monitoring temperature variations around the nasal area, which are related to inhalation and exhalation; and the facial skin temperature can be easily determined from thermal images simultaneously.
The circulation of blood in the face causes tiny color changes providing a visible facial image that can be used to measure heart rate. In addition, the IRT system displays the result "Infection" or "Healthy" within 10 seconds using the discriminant logistic regression func-tion, which bases the output on heart rate, respiratory rate, and facial skin temperature [24].

Another work has been based on this type of camera to measure the heart activity, a new camerabased vital sign estimation algorithm that meets the challenges of darker skin tones, under low light conditions and/or during the movement of a person in front of the camera. The Distance presents a new method of combining skin color change signals from different regions of the face using a weighted average, where the weights depend on blood perfusion and the incident light intensity in the region, in order to improve the signal-to-noise ratio (SNR) of a camerabased estimate. The contribution of this work is to developing a formal method to take into account changes in blood perfusion and incident light intensity in different regions of the face to improve the precision of vital sign estimation in demanding situations (e.g., dark skin tones and/or poor lighting conditions). In a further development, they proposed a new motion-tracking algorithm that tracks different facial regions separately, thus improving the performance of vital sign estimation in scenes of natural motion such as viewing content on a computer screen, playing a video and talking on Skype [25]. 
In order to integrate all monitoring systems into a single system that will monitor patients in critical cases and especially all people contaminated by the Covid-19 pandemic, we need a prototype that contains heterogeneous (CPU-GPU and CPU-FPGA) and homogenous (CPU, FPGA...) systems and a ventilation system that will help people to ensure good breathing. In this way our proposed system consists of a camera and a ventilation system that helps patients get enough oxygen and monitoring of heart rate, as well as a thermal camera that will monitor the temperature.

The use of the camera in place of the $\mathrm{O} 2$, temperature, and ECG sensors will decrease the frequent contact with patients infected with the Covid-19 virus. Traditional methods used in respiratory rate, heart rate, and temperature monitoring are based on sensors with direct contact between the patient and the sensor used, which requires sterilization before each use for reusable sensors. On the other hand, the single-use sensor will create losses for each use on the hardware side.

This system will eliminate the need to use the $\mathrm{O} 2$ and $\mathrm{CO} 2$ and temperature sensors each time to deliver the right amount of oxygen, so we allow for accurate patient temperature monitoring. The RGB camera will monitor the patient's face to raise the heart rate based on the color change, if it detects weak breathing or heartbeat the system will trigger an alarm for the doctor and the ventilator controlled by an electric motor in real-time. The thermal camera will focus on all the patient's body to raise the temperature of the patients, the use of this camera will allow us to have the temperature status of the patient because among the consequences of the Covid-19 virus the temperature increase.

The system proposed in this paper is based on the combination of embedded systems and biomedical engineering to have an embedded medical device that offers an inventive solution for real-time monitoring of patients contaminated by Covid-19. The focus on respiratory, cardiac and temperature monitoring is due to the consequences of the Covid-19 Virus which directly contaminates the respiratory system creating problems in breathing and temperature increase. For this reason, the most critical parameter to be monitored in real-time is a respiratory rate, heart rate, and temperature. After the extraction of the different signs such as temperature, heart rate, and respiratory rate it is necessary to apply compression techniques, to reduce the size of the file in order to send it to the database [26].

\section{CONCLUSIONS}

Monitoring of vital signs such as heart rate and respiratory rate as well as temperature is a very important step for good diagnosis of patients. In this period and with the Covid-19 pandemic we need noncontact monitoring techniques to protect doctors from contamination. Integration of embedded systems in the biomedical field especially patient monitoring based on non-contact techniques. Embedded systems with their variation either heterogeneous (CPU-GPU, CPU-FPGA) or homogeneous (CPU, GPU, FPGA...) will give flexibility in terms of speed and real-time. The implementation of vital signs monitoring algorithms in workstations and their validation using tools such as MATLAB does not give the hand to a real case study, for this reason, it is necessary to work on real patient cases. In this work, we have treated the different proposed work based on different imaging sensors such as thermal and RGB cameras.

\section{ACKNOWLEDGEMENTS}

We owe a debt of gratitude to the Ministry of National Education, Vocational Training, Higher Education and Scientific Research (MENFPESRS) and National Centre for Scientific and Technical Research of Morocco (CNRST) for its financial support for the project Cov/2020/109.

\section{REFERENCES}

R. B. Duffey and E. Zio, "Analysing Recovery From Pandemics by Learning Theory: The Case of CoVid19," in IEEE Access, vol. 8, pp. 110789-110795, 2020.

J. Zhang et al., "Navigating the Pandemic Response Life Cycle: Molecular Diagnostics and Immunoassays in the Context of COVID-19 Management," in IEEE Reviews in Biomedical Engineering.

V. Chamola, V. Hassija, V. Gupta and M. Guizani, "A Comprehensive Review of the COVID-19 Pandemic and the Role of IoT, Drones, AI, Blockchain, and 5G in Managing its Impact," in IEEE Access, vol. 8, pp. 90225-90265.

X. Ding et al., "Wearable Sensing and Telehealth Technology with Potential Applications in the Coronavirus Pandemic," in IEEE Reviews in Biomedical Engineering.

R. B. Duffey and E. Zio, "Analyzing Recovery From Pandemics by Learning Theory: The Case of CoVid19," in IEEE Access, vol. 8, pp. 110789-110795, 2020.

B. Hu, S. Wei, D. Wei, L. Zhao, G. Zhu and C. Liu, "Multiple Time Scales Analysis for Identifying 
Congestive Heart Failure Based on Heart Rate Variability," in IEEE Access, vol. 7, pp. 17862-17871, 2019.

D. Biswas, N. Simões-Capela, C. Van Hoof and N. Van Helleputte, "Heart Rate Estimation From Wrist-Worn Photoplethysmography: A Review," in IEEE Sensors Journal, vol. 19, no. 16, pp. 6560-6570, 15 Aug.15, 2019.

V. L. Petrović, M. M. Janković, A. V. Lupšić, V. R. Mihajlović and J. S. Popović-Božović, "High-Accuracy Real-Time Monitoring of Heart Rate Variability Using $24 \mathrm{GHz}$ Continu-ous-Wave Doppler Radar," in IEEE Access, vol. 7, pp. 74721-74733, 2019.

S. Kontaxis et al., "ECG-Derived Respiratory Rate in Atrial Fibrillation," in IEEE Transac-tions on Biomedical Engineering, vol. 67, no. 3, pp. 905-914, March 2020

C. Uysal and T. Filik, "RF-Based Noncontact Respiratory Rate Monitoring With Parametric Spectral Estimation," in IEEE Sensors Journal, vol. 19, no. 21, pp. 98419849, 1 Nov.1, 2019.

Hertzman, A.B. Observations on the finger volume pulse recorded photoelectrically. Am. J. Physiol. 1937, 119, 334-335.

Wang, W.; den Brinker, A.C.; Stuijk, S.; de Haan, G. Algorithmic principles of remote PPG. IEEE Trans. Biomed. Eng. 2017, 64, 1479-1491.

S. Sanyal and K. K. Nundy, "Algorithms for Monitoring Heart Rate and Respiratory Rate From the Video of a User's Face," in IEEE Journal of Translational Engineering in Health and Medicine, vol. 6, pp. 1-11, 2018, Art no. 2700111.

Khanam, F.T.Z.; Al-Naji, A.; Chahl, J. Remote Monitoring of Vital Signs in Diverse Non-Clinical and Clinical Scenarios Using Computer Vision Systems: A Review. Appl. Sci. 2019, 9, 4474.

Dalal, H.; Basu, A.; Abegaonkar, M.P. Remote sensing of vital sign of human body with radio frequency. CSI Trans. ICT 2017, 5, 161-166.

Ghafouri-Shiraz, H. Ultra-wide patch antenna array design at $60 \mathrm{GHz}$ band for remote vital sign monitoring with Doppler radar principle. J. Infrared Millim. Terahertz Waves 2017, 38, 548-566.

Yang, M.; Liu, Q.; Turner, T.; Wu, Y. Vital sign estimation from passive thermal video. In Proceedings of the 2008 IEEE Conference on Computer Vision and Pattern Recognition, Anchorage, AK, USA, 23-28 June 2008; pp. 1-8.

Bennett, S.; El Harake, T.N.; Goubran, R.; Knoefel, F. Adaptive Eulerian Video Processing of Thermal Video: An Experimental Analysis. IEEE Trans. Instrum. Meas. 2017, 66, 2516-2524.

Nakajima, K.; Osa, A.; Miike, H. A method for measuring respiration and physical activity in bed by optical flow analysis. In Proceedings of the 19th Annual International Conference of the IEEE Engineering in Medicine and Biology Society. 'Magnificent Milestones and Emerging Opportunities in Medical Engineering' (Cat. No. 97CH36136), Chicago, IL, USA, 30 October-2 November 1997; pp. 2054-2057. 50 .
Frigola, M.; Amat, J.; Pagès, J. Vision based respiratory monitoring system. In Proceedings of the 10th Mediterranean Conference on Control and Automation (MED 2002), Lisbon, Portugal, 9-12 July 2002; pp. 9 13.

Al-Naji, A.; Chahl, J. Remote respiratory monitoring system based on developing motion magnification technique. Biomed. Signal Process. Control 2016, 29. $1-10$.

Latif Rachid, Saddik Amine, "Embedded Implementation of Biomedical Applications in Het-erogeneous Systems", Biomedical Spectroscopy and Imaging, vol. 8, no. 3-4, pp. 73-80, 2019.

R. Latif, A. Saddik, A. Elouardi, "Evaluation of Agricultural Precision Algorithms on UAV Images," 2019 International Conference of Computer Science and Renewable Energies (ICCSRE), Agadir, Morocco, 2019, pp. 1-4, doi: 10.1109/ICCSRE.2019.8807604

Guanghao Sun, Yosuke Nakayama, Sumiyakhand Dagdanpurev, Shigeto Abe, Hidekazu Nishimura, Tetsuo Kirimoto, Takemi Matsui, "Remote sensing of multiple vital signs using a CMOS camera-equipped infrared thermography system and its clinical application in rapidly screening patients with suspected infectious diseases", International Journal of Infectious Diseases, Vol. 55, 2017, pp. 113-117.

Mayank Kumar, Ashok Veeraraghavan, and Ashutosh Sabharwal, "DistancePPG: Robust non-contact vital signs monitoring using a camera," Biomed. Opt. Express 6, 1565-1588 (2015).

R. Latif, F. Z. Guerrouj, A. Saddik and O. El B'Charri, "ECG signal compression based on ASCII coding using CUDA architecture," 2019 4th World Conference on Complex Systems (WCCS), Ouarzazate, Morocco, 2019, pp. 1-6, doi: 10.1109/ICoCS.2019.8930744. 